\title{
Durabilidad de la madera \\ de Prosopis laevigata y efecto de sus \\ extractos en hongos que degradan la madera
}

\author{
Durability of wood of Prosopis laevigata and the effect \\ of its extractives on wood-decaying fungus
}

\author{
Artemio Carrillo-Parra1, František Hapla2, Carsten Mai2 \\ y Fortunato Garza-Ocañas 1
}

\begin{abstract}
RESUMEN
Se determinó la durabilidad natural de la madera de Prosopis laevigata de cuatro sitios del Noreste de México mediante a) microcosmos terrestres (Pre-norma Europea 807) en un suelo tipo vertisol durante 32 semanas y $b$ ) en condiciones de laboratorio (Norma Europea 113 modificada) al exponer la madera a los hongos Coniophora puteana, Irpex lacteus, Pleurotus ostreatus y Trametes versicolor durante 16 semanas. La durabilidad natural fue cuantificada como pérdida de masa y pérdida de módulo de elasticidad dinámico $\left(\mathrm{MOE}_{\mathrm{din}}\right)$. Adicionalmente, se determinó el efecto en el crecimiento de los hongos $C$. puteana y $T$. versicolor producido por los extractos de la madera de $P$. laevigata obtenidos por agua caliente, etanol y acetona en concentraciones de 100 partes por millón (ppm), 1000 ppm, 5000 ppm y 10000 ppm. Los resultados indican que $P$. laevigata es altamente resistente en el microcosmos terrestre, con pérdidas en masa de $(9 \pm 2) \%$ a $(17 \pm 5) \%$ y $\mathrm{MOE}_{\text {din }}$ de $(20 \pm 4) \%$ a $(39 \pm 11) \%$. En el segundo método, la pérdida en masa producida por los cuatro hongos fue de $(0,7 \pm 0,5) \%$ a $(2,6 \pm 3,2) \%$ y clasificada según la Norma Europea 350-1 como clase 1 (muy durable). La pérdida del $\mathrm{MOE}_{\text {din }}$ fue de $(3,8 \pm 4,2) \%$ a $(19,9 \pm 5,6) \%$. Los extractos de $P$. laevigata obtenidos con agua caliente en concentración de 10000 ppm mostraron el mayor porcentaje de inhibición al reducir el crecimiento de C. puteana $(83 \pm 8) \%$ y $(93 \pm 6) \%$ para $T$. versicolor.
\end{abstract}

PALABRAS CLAVE:

Durabilidad natural, extractos, Prosopis laevigata, pudrición.

\begin{abstract}
The natural durability of mezquite wood (Prosopis laevigata) from four localities in Northeast Mexico was determined using two test, a) terrestrial microcosm (European pre-norm 807) during 32 weeks on a non sterilized vertisol soil and, $b$ ) laboratory conditions according to the modify European Norm 113, the wood was exposed by 16 weeks to Basidiomycetes Trametes versicolor, Coniophora puteana, Irpex lacteus and Pleurotus ostreatus. The natural durability of two methods were determined by mass loss and by the dynamic modulus of elasticity loss $\left(\mathrm{MOE}_{\text {dyn }}\right)$. Additionally the inhibition effect of wood extracts obtained from wood sawdust in either hot water, ethanol or acetone at 100 parts per million (ppm), 1000 ppm, 5000 ppm and 10000 ppm on growth of C. puteana and T. versicolor was also determined. The results showed that $P$. laevigata wood from the four sites is similar and very resistant to soil contact; the mass lost was ranging from $(9 \pm 2) \%$ to $(17 \pm 5) \%$. The $\mathrm{MOE}_{\text {din }}$ lost of $P$.
\end{abstract} Linares, Nuevo León, México. C.e.: arte_carr@hotmail.com 
laevigata was ranging from $(20 \pm 4) \%$ to (39 \pm 11$) \%$, the control species showed values of $(91 \pm 13) \%$ and $(62 \pm 15) \%$ respectively. The second method (modify european norm 113) showed mass loss of $P$. laevigata for the four localities and the four fungi ranging from $(0,7 \pm 0,5) \%$ to $(2,6 \pm 3,2) \%$, placed this species as a class 1 (i.e. very durable) according to the European Norm 350-1. The MOE losses was from $(3,8 \pm 4,2) \%$ to $(19,9 \pm$ $5,6) \%$. The hot water extracts at 10000 ppm concentration inhibited growth of $C$. puteana and $T$. versicolor by $(83 \pm 8) \%$ and $(93 \pm 6) \%$ respectively.

KEY WORDS:

Natural durability, extractives, Prosopis laevigata, decay.

\section{INTRODUCCIÓN}

El género Prosopis comprende alrededor de 44 especies y se encuentra ampliamente distribuido en zonas áridas $y$ semiáridas del mundo en condiciones de suelo pobre (Burkart, 1976; Alden, 1995; Juárez et al., 2002). Se localizan en África, Asia y América (Pasiecznik et al., 2001). En Norteamérica existe el complejo denominado México-Texano donde se desarrollan diez especies en forma natural; Sudamérica, principalmente en Argentina y en los países vecinos, se considera el centro de dispersión del continente Americano del género Prosopis (Burkart, 1976; INE, 1994).

La madera de Prosopis se utiliza principalmente para la producción de carbón, por su alta densidad y la forma irregular en que se desarrollan los fustes. En menor escala se utiliza para la producción de muebles, mangos para herramientas y duelas (Pasiecznik et al., 2001).

En estudios de durabilidad natural, se ha clasificado la madera de algunas especies de Prosopis como durables (Wolf y Perales, 1985). Durabilidad natural de la madera es entendida como la resistencia que presentan algunas especies contra el deterioro ambiental que se puede manifestar como pudrición (Balaban et al., 2003). La durabilidad, el color, el olor, la permeabilidad y la reducción en contracción e hinchamiento de cada especie maderera, se atribuye a la presencia de los extractos (Hillis, 1968; Hillis, 1987; Reyes-Chilpa et al., 1998; Aloui et al., 2004; Gutiérrez et al., 2006). Los extractos son compuestos químicos que constituyen de $2 \%$ a $10 \%$ del peso seco en especies de clima templado y hasta $20 \%$ en maderas de clima tropical (Fengel y Wegener, 1989; Mantanis et al., 1995) y pueden inducir la durabilidad en la madera.

La madera de especies como Tectona grandis, Chamaecyparis nootkatensis, Thuja plicata, presentan una alta resistencia al deterioro causado por insectos y hongos; debido a la presencia de compuestos químicos como las tectoquinonas $y$ quinonas (Haupt et al., 2003; Taylor et al., 2006; Thulasidas y Bhat, 2007).

El uso de $P$. laevigata se puede ampliar si se analiza detalladamente su durabilidad natural, característica que ha sido poco estudiada (Donoso et al., 1984; Rodríguez y Maldonado, 1996; Ríos et al., 2001; Géardin et al., 2004). Esto posibilitaría su uso en condiciones a la intemperie como recubrimientos de pisos, ventanas y muebles de jardín donde se requiere alta durabilidad (Folliott $y$ Thames, 1983; INE, 1994; Meraz et al., 1998; Giménez et al., 2000).

Por lo tanto, determinar la durabilidad natural de la madera de $P$. laevigata, la eficiencia de sus extractos en inhibir el crecimiento de hongos degradadores de la madera, identificar y finalmente utilizar los compuestos químicos que producen esta resistencia en maderas con baja resistencia, son líneas de investigación que incrementarían la utilización de esta especie. 


\section{OBJETIVOS}

Los objetivos de esta investigación son: 1) determinar y clasificar la durabilidad natural de la madera de $P$. laevigata proveniente de cuatro sitios al ser expuesta a microcosmos terrestres y en laboratorio a los hongos $C$. puteana, I. lacteus, $P$. ostreatus y $T$. versicolor, 2) establecer, en condiciones de laboratorio, el efecto de los extractos de la madera de $P$. laevigata en el crecimiento de los hongos $C$. puteana y $T$. versicolor, degradadores de la madera.

\section{METODOLOGÍA}

La durabilidad natural de la madera de $P$. laevigata fue determinada con probetas provenientes de 12 árboles extraídos en el estado de Nuevo León, de los sitios Rancho Saltilleros municipio de China, Rancho San Lorenzo, municipio de General Terán, Ejido La Reforma, municipio de Linares y Ejido Santa Gertrudis, municipio de Dr. Arroyo. De cada sitio se obtuvo información relacionada con las condiciones ambientales, incluyendo temperatura, precipitación y localización geográfica (Tabla 1). En forma adicional y para cumplir con los requisitos de las normas empleadas, se usaron probetas testigo de la madera de albura de Pinus sylvestris y Fagus sylvatica.

\section{Durabilidad natural de la madera en microcosmos terrestre (Pre-norma Europea 807)}

La durabilidad en microcosmos terrestre se determinó siguiendo la Pre-norma Europea 807 (ENV 807, 2001). El procedimiento consistió en seleccionar de cada sitio 20 probetas con dimensiones de 100 $\mathrm{mm} \times 10 \mathrm{~mm} \times 5 \mathrm{~mm}$ en dirección longitudinal, radial y tangencial, respectivamente, de madera de $P$. laevigata; además, se utilizaron 20 probetas de madera de $P$. sylvestris y 20 de $F$. sylvatica las cuales sirvieron como especies testigo. Las 120 probetas fueron colocadas al azar en dos recipientes de plástico $(32 \mathrm{~cm} \times 40 \mathrm{~cm}$ $x 22 \mathrm{~cm}$ ) conteniendo microcosmos terrestre. Cada probeta se colocó a $2 \mathrm{~cm}$ de distancia y se enterraron $8 \mathrm{~cm}$. El suelo se mantuvo a un contenido de humedad en un rango de $20 \%$ a $60 \%$ de su máxima capacidad de campo

Tabla 1. Localización geográfica, temperatura y precipitación de los sitios donde se extrajeron los árboles para la elaboración de las probetas de madera de $P$. laevigata.

\begin{tabular}{lcccc}
\hline Descripción & China & General Terán & Linares & Dr. Arroyo \\
\hline Localidad & Rancho & Rancho & Ejido & Ejido Santa \\
& Saltilleros & San Lorenzo & La Reforma & Gertrudis \\
\hline & $25^{\circ} 24^{\prime} 23^{\prime \prime}$ & $25^{\circ} 20^{\prime} 18^{\prime \prime}$ & $24^{\circ} 42^{\prime} 05^{\prime \prime}$ & $23^{\circ} 54^{\prime} 48^{\prime \prime}$ \\
Latitud & $99^{\circ} 10^{\prime} 22^{\prime \prime}$ & $99^{\circ} 31^{\prime} 00^{\prime \prime}$ & $99^{\circ} 32^{\prime} 05^{\prime \prime}$ & $100^{\circ} 10^{\prime} 14^{\prime \prime}$ \\
Longitud & $22-24$ & $22-24$ & $20-22$ & $16-20$ \\
Temperatura ${ }^{\circ} \mathrm{C}^{*}$ & 512 & 631 & 759 & $300-600$ \\
Precipitación $(\mathrm{mm})^{*}$ & & & & \\
\hline
\end{tabular}

* Fuente: INEGI (2000) 
durante 32 semanas. La durabilidad natural se cuantificó como el grado de deterioro de la madera mediante la pérdida de masa y la pérdida del módulo de elasticidad dinámico $\mathrm{MOE}_{\text {din }}$, los procedimientos se detallan a continuación:

\section{Pérdida de masa}

Este procedimiento consistió en determinar la reducción de masa de las probetas de madera expuestas a microcosmos terrestres, al obtener la diferencia en porciento de la masa de madera en condición seca al inicio del experimento y la masa encontrada después de 32 semanas. La pérdida de masa fue determinada mediante la siguiente fórmula:

$$
P \cdot M=\frac{M_{i}-M_{f}}{M_{i}}
$$

Donde:

$P . M=$ Pérdida de masa $(\%)$

$M_{i}=$ masa anhidra de la probeta al inicio del ensayo $(\mathrm{g})$

$M_{f}=$ masa anhidra de la probeta al final del ensayo (g)

\section{Pérdida de $M O E_{\text {din }}$}

Este procedimiento fue establecido por Machek et al. (2001), tiene la ventaja de no ser destructivo y no requiere de instalar algún equipo (Hearmon, 1966; Machek et al., 1998; Machek et al., 2001). Se utiliza para cuantificar la pudrición en probetas de madera, debido a que la pérdida del $\mathrm{MOE}_{\text {din }}$ es mucho más sensible que otras pruebas y presenta valores altamente significativos, incluso en etapas iniciales de deterioro cuando la pérdida de masa es aún baja.
El $\mathrm{MOE}_{\text {din }}$ se calculó en probetas a punto de saturación de la fibra, mediante la inducción de vibraciones producidas por el golpe de un pequeño martillo en el punto medio de la probeta. La señal se registró con un transductor colocado a un extremo de la probeta a una distancia de 0,224 veces su longitud, el cual captura las frecuencias y las envía hacia el equipo GrindoSonic (Lemmers, Belgium) que muestra los valores de frecuencia en $\mathrm{kHz}$. Los valores fueron registrados al inicio del ensayo, y posteriormente en las semanas 8, 16, 24 y 32. La información obtenida fue utilizada en las siguientes fórmulas para determinar el $\mathrm{MOE}_{\mathrm{din}}$ :

$$
\begin{gathered}
M O E_{d i n}=\frac{4 \cdot \pi^{2} \cdot l^{4} \cdot f^{2} \cdot \rho \cdot \mathrm{A}}{m_{1}^{4} \cdot I} \cdot\left(1+\frac{I}{l^{2} \cdot A} \cdot K_{1}\right) \\
I=\frac{b \cdot a^{2}}{12}
\end{gathered}
$$

Donde:

$M O E_{\text {din }}=$ Módulo de elasticidad dinámico $\left(\mathrm{N} / \mathrm{mm}^{2}\right)$

$I$ = Momento de inercia $\left(\mathrm{mm}^{4}\right)$

$A=$ Área de sección transversal $\left(\mathrm{mm}^{2}\right)$

$f=$ Frecuencia $(\mathrm{kHz})$

rho $=$ Densidad $\left(\mathrm{g} / \mathrm{mm}^{3}\right)$

I = Longitud de la probeta $(\mathrm{mm})$

$K_{1}=$ Constante $(49,48)$

$m_{1}=$ Constante $(4,72)$

$a=$ grosor de la probeta $(\mathrm{mm})$

$b=$ ancho de la probeta $(\mathrm{mm})$

\section{Durabilidad de la madera de $P$. laevigata contra Basidiomycetes}

La durabilidad natural de la madera de $P$. laevigata con respecto a la pudrición ocasionada por los hongos C. puteana, I. lacteus, $P$. ostreatus y $T$. versicolor, se determinó de acuerdo con la Norma Europea 113 modificada (EN 113, 1996). La modificación consistió en cambiar el 
tamaño de las probetas a $100 \mathrm{~mm} \times 10$ $\mathrm{mm} \times 5 \mathrm{~mm}$ en dirección longitudinal, radial y tangencial respectivamente. Los porcentajes de pérdida de masa y la pérdida del $\mathrm{MOE}_{\text {din }}$ fueron utilizadas como indicadores de la durabilidad.

Para determinar la pérdida de masa y la pérdida del $\mathrm{MOE}_{\text {din }}$ producida por cada hongo en la madera de $P$. laevigata se utilizaron 9 probetas de $P$. laevigata por sitio; es decir, se utilizaron un total de 36 probetas de esta especie; como especie testigo se utilizaron 12 probetas de F. sylvatica y para conocer el grado de colonización o virulencia de cada hongo se seleccionaron 24 probetas de F. sylvatica. El efecto de lixiviación, que es el arrastre de compuestos producido en la madera por la humedad ambiental, se midió al colocar 12 probetas de la madera de $P$. laevigata de cada sitio y 12 probetas de F. sylvatica en el medio de cultivo.

La totalidad de las probetas se esterilizó en una autoclave (Tuttnauer Systec Tipo 5075 ELVC, Wettenberg, Alemania) durante 20 minutos, a una temperatura de $121{ }^{\circ} \mathrm{C}$. Las probetas se colocaron en frascos de cultivo con $50 \mathrm{ml}$ de maltaagar solidificado; posteriormente, en cada frasco se colocó un inóculo de $1 \mathrm{~cm}$ del hongo y ocho probetas de madera. De las ocho probetas de cada frasco, seis correspondían a $P$. laevigata, las cuales fueron seleccionadas al azar de los cuatro sitios y dos probetas de F. sylvatica. Los frascos con las muestras se incubaron en un cuarto de cultivo con ambiente controlado, por un periodo de 16 semanas, a una temperatura de $20 \pm 2{ }^{\circ} \mathrm{C}$ y una humedad relativa $(\mathrm{HR}) \mathrm{de}$ $65 \pm 5 \%$.

Al término del ensayo, se midió la pérdida de masa y la pérdida de $\mathrm{MOE}_{\text {din }}$ de las probetas con el equipo GrindoSonic (Lemmers, Belgium). Los valores obtenidos se ajustaron al restar la pérdida en masa que se ocasionó por el efecto de lixiviación de los compuestos de la madera y que no se le puede atribuir a la degradación del hongo.

\section{Inhibición en el crecimiento de los hongos provocado por los extractos de la madera}

Para determinar el efecto inhibitorio en el crecimiento de los hongos degradadores de la madera $C$. puteana y $T$. versicolor provocado por los extractos de la madera de $P$. laevigata, se sometió el aserrín de $P$. laevigata a la extracción en aparatos Soxhlet, en una estufa de aceite, a una temperatura de $135{ }^{\circ} \mathrm{C}$, se utilizaron como solventes extractivos agua caliente, etanol y acetona. La extracción se realizó al completar 24 ciclos en un periodo de seis horas; posteriormente, el solvente se eliminó con un evaporador de vacío (Rotavapor Tipo R-114 Büchi, Essen, Alemania) a una temperatura de $40{ }^{\circ} \mathrm{C}$.

Una vez obtenidos los extractivos, se diluyeron en acetona a concentraciones de 100 ppm, 1000 ppm, 5000 ppm y 10000 ppm para facilitar la disolución y homogeneizar las condiciones en el manejo de todos los extractos. La mezcla se agitó con un mezclador Heidolph Tipo Mr 3003 (Heidolph Elektro $\mathrm{GmbH}$, Kelheim, Alemania) por 10 minutos antes de ser colocados en el medio de cultivo. Para preparar el medio de cultivo se utilizaron $20 \mathrm{ml}$ de maltaagar en cajas Petri de $90 \mathrm{~mm}$ de diámetro.

Al solidificarse la mezcla de acetona con los solventes y el extracto de maltaagar, se aplicó el inóculo con un sacabocado de $10 \mathrm{~mm}$ de diámetro proveniente de los márgenes externos de las colonias activas en crecimiento de $C$. puteana (hongo de pudrición café) y $T$. versicolor (hongo de pudrición blanca). Para cada 
concentración de extracto y especie de hongo, se colocaron cinco repeticiones, obteniendo un total de 80 cajas Petri. Se prepararon adicionalmente 10 cajas Petri para cada hongo con medio de cultivo (malta-agar) a las cuales no se les aplicaron extractos (testigo). Para determinar la influencia de la acetona en el desarrollo de los hongos, se prepararon adicionalmente 5 cajas Petri para cada hongo y se les añadieron $10 \mathrm{ml}$ de acetona.

Los cultivos se mantuvieron en una cámara de crecimiento a una temperatura de $20 \pm 2{ }^{\circ} \mathrm{C}$ y un contenido de humedad del $65 \pm 5 \%$, hasta que el micelio del testigo alcanzó la orilla de la caja Petri. La inhibición del crecimiento se determinó de acuerdo con Géardin et al. (2004), donde el efecto se calcula con la siguiente fórmula:

$$
G i=100\left(1-\frac{d_{1}}{d_{0}}\right)
$$

Donde:

$G i=\begin{gathered}\text { Inhibición del crecimiento en } \\ \text { porciento. }\end{gathered}$ $d_{1}=$ Diámetro promedio del cultivo en presencia de extractos ( $\mathrm{mm}$ ).

$d_{0}=$ Diámetro promedio del cultivo testigo (mm).

\section{RESULTADOS Y DISCUSIÓN}

\section{Durabilidad natural de la madera en microcosmos terrestre (Pre-norma Europea 807)}

\section{Pérdida de masa}

La madera de $P$. laevigata en contacto con microcosmos terrestre presentó una alta durabilidad contra los microorganismos degradadores de la madera, según se aprecia en la figura 1. La menor pérdida de masa se observó en los sitios
China, General Terán y Linares con valores de $10 \pm 1 \%, 9 \pm 2 \%$ y $12 \pm 1 \%$ respectivamente, mientras que en el sitio Dr. Arroyo, ubicado geográficamente más al norte, con rango de temperatura y precipitación más bajo que los tres sitios antes mencionados, la pérdida en masa fue mayor (17 $\pm 5 \%)$. Los valores de pérdida en masa presentados en los diferentes sitios fueron menores que los testigos $P$. sylvestris y $F$. sylvatica, cuyos valores fueron de $35 \pm 7) \%$ y $84 \pm 10 \%$, respectivamente.

\section{Pérdida en $M O E_{\text {din }}$}

La reducción en el $\mathrm{MOE}_{\text {din }}$, resultado del deterioro producido en la estructura de la madera por los organismos presentes en el microcosmos terrestre, presentó los porcentajes más bajos en los sitios China, General Terán y Linares con $20 \pm 4 \%$, $22 \pm 8 \%$ y $21 \pm 7 \%$, respectivamente (Figura 1). En el sitio Dr. Arroyo se observaron valores de $\mathrm{MOE}_{\text {din }}$ relativamente altos, con un promedio de $39 \pm 11 \%$, sin embargo, el $\mathrm{MOE}_{\text {din }}$ se consideró bajo al compararse con las especies testigo, $P$. sylvestris, cuya pérdida promedió $62 \pm 15 \%$ y $F$. sylvatica, que tuvo un valor de $91 \pm 13 \%$.

La utilidad de usar la pérdida de $\mathrm{MOE}_{\text {din }}$, como herramienta para la detección temprana de la degradación ocasionada por el microcosmos terrestre, se puede comprobar en la figura 2, al observar que ya para la semana 8, el testigo F. sylvatica mostró pérdida de $\mathrm{MOE}_{\text {din }}$ de $64 \pm 20 \%$, valor considerado alto. En $P$. sylvestris, la pérdida fue de $19 \pm 6 \%$ y la menor pérdida se presentó en la madera de $P$. laevigata de los diferentes sitios con porcentajes de $3 \pm 3 \%$ a $9 \pm 6 \%$.

En la semana 16, la pérdida de $\mathrm{MOE}_{\text {din }}$ observada en $F$. sylvatica fue de $79 \pm 22 \%$, para $P$. sylvestris fue de $40 \pm$ $9 \%$. Para $P$. laevigata, el sitio con mayor pérdida fue: Dr. Arroyo con $24 \pm 10 \%$, 


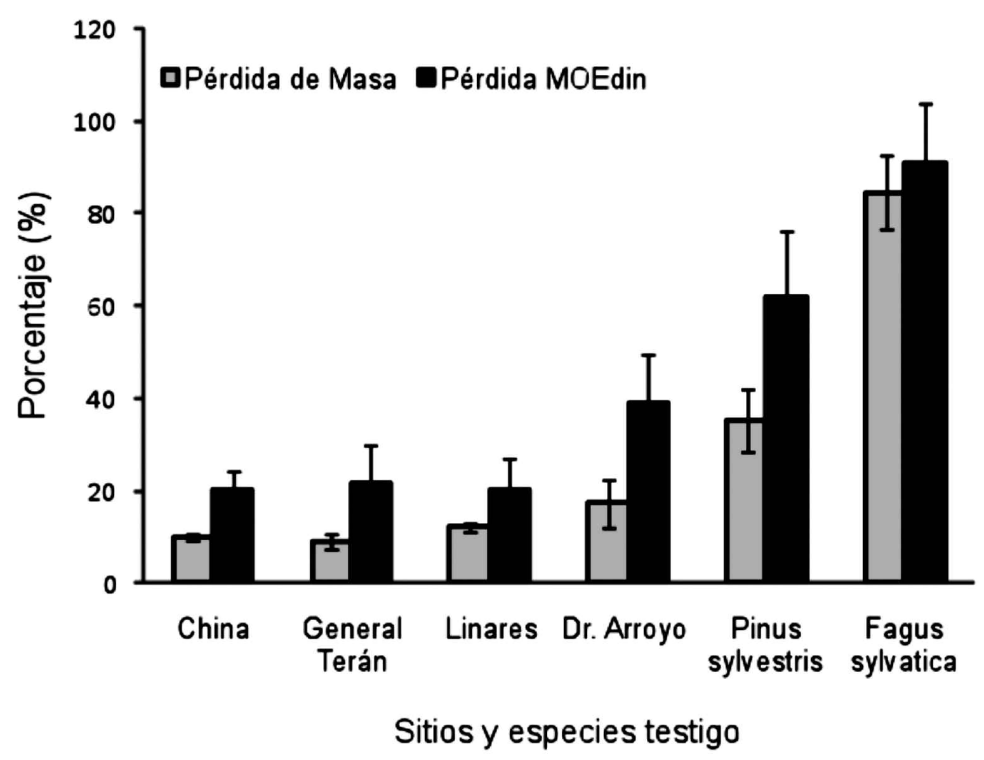

Figura 1. Pérdida de masa y módulo de elasticidad dinámico $\left(\mathrm{MOE}_{\mathrm{dyn}}\right)$ presentado por la madera de $P$. laevigata y los testigos $P$. sylvestris y $F$. sylvatica después de 32 semanas de exposición a microcosmos terrestre.

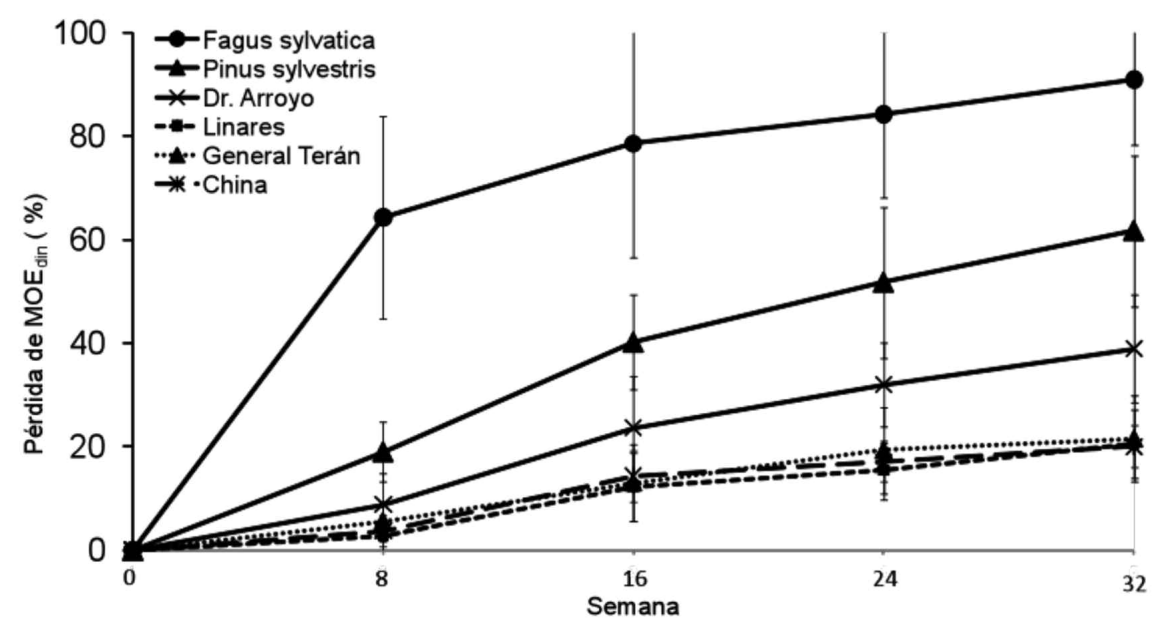

Figura 2. Pérdida de $\mathrm{MOE}_{\text {din }}$ en las semanas $8,16,24$ y 32 de $P$. laevigata y de los testigos $P$. sylvestris y $F$. sylvatica expuestos a microcosmos terrestre. 
en los sitios China, General Terán y Linares, el porcentaje varió de $12 \pm 6 \%$ a $14 \pm 5 \%$, valores que se consideran bajos en comparación con las especies testigo. Posteriormente, la pérdida de $\mathrm{MOE}_{\text {din }}$ aumentó paulatinamente, en la semana 32 la pérdida de los testigos $P$. sylvestris y $F$. sylvatica fue de $62 \pm 15 \%$ y $91 \pm 13 \%$, respectivamente. El sitio $\mathrm{Dr}$. Arroyo mostró la mayor pérdida para $P$. laevigata con $39 \pm 11 \%$, en los sitios China, Linares y General Terán, la pérdida fue de $20 \pm 4 \%, 20 \pm 7 \%$ y $22 \pm 8 \%$, respectivamente. La temprana detección de pérdida del $\mathrm{MOE}_{\text {din }}$ mostrada por este método y observada ya para la semana 16 , se debe a que los microorganismos presentes en el microcosmos terrestre inician la degradación de algunos componentes de la madera como la celulosa que le proporciona estabilidad mecánica.

\section{Durabilidad de la madera de $P$. laevigata contra Basidiomycetes}

\section{Pérdida en masa \\ Las probetas de $P$. laevigata provenientes de cuatro sitios expuestas a los hongos: $P$. ostreatus, I. lacteus, C. puteana y $T$. versicolor, presentaron pérdidas en masa de $0,7 \pm 0,5 \%$, a $2,6 \pm 3,2 \%$ después de restar $1,68 \%$ que corresponde a la pérdida de masa ocasionada por la lixivia- ción. Según la Norma Europea EN 113 considera válido un ensayo de degrada- ción producida por hongos, cuando la pérdida de masa para la especie testigo de F. sylvatica es mayor a $12 \%$.}

El hongo $P$. ostreatus redujo la masa del testigo en $42 \pm 7,5 \%$, seguido por I. lacteus y $T$. versicolor con $50 \pm 5,4 \%$ y $51 \pm 20 \%$, respectivamente. La mayor pérdida de masa la produjo $C$. puteana con $63 \pm 2 \%$ (Figura 3 ). Por lo anterior, se puede inferir que la prueba realizada es válida, al observar que la infección de los hongos utilizados fue la adecuada.

La durabilidad mostrada por la madera de $P$. leavigata contra los cuatro hongos ensayados, se puede observar en la figura 3. Las mayores pérdidas en masa se presentaron en la localidad China al obtener valores de $2,1 \pm 0,4 \%$, $2,5 \pm 0,3 \%, 2,3 \pm 0,6 \%$ y $2,2 \pm 0,3 \%$, para los hongos $C$. puteana, I. lacteus, $P$. ostreatus y $T$. versicolor, respectivamente. En el sitio General Terán se observaron pérdidas de $1,6 \pm 0,5 \%, 1,5 \pm 0,6 \%$, $1,6 \pm 0,7 \%$ y $1,6 \pm 0,4 \%$ para las mismas especies. En Linares el porcentaje de afectación fue de $1,1 \pm 0,5 \%, 2,5 \pm 3,2 \%$, $1,2 \pm 0,8 \%$ y $1,1 \pm 0,4 \%$. La menor pérdida en masa se presentó en el sitio de Dr. Arroyo con valores de $0,7 \pm 0,6 \%$, $1,4 \pm 0,6 \%, 0,7 \pm 0,5 \%$ y $0,9 \pm 0,5 \%$ para las especies C. puteana, I. lacteus, $P$. oetreatus y $T$. versicolor, respectivamente.

Según la pérdida de masa y de acuerdo con el Sistema de Clasificación EN 350-1 (EN 350-1, 1994), la madera de $P$. laevigata es clasificada con una durabilidad clase 1 (madera de alta durabilidad con pérdida de masa $\leq 5 \%$ ) para todos los sitios estudiados.

La resistencia a la degradación de la madera de $P$. laevigata expuesta al hongo T. versicolor (valor máximo de $2,2 \pm 0,3 \%$ ) es mayor a la reportada por Wolf y Perales (1985), para la especie Prosopis glandulosa, ya que ellos encontraron pérdidas en masa de $4 \%$ después de exponer la madera por 12 semanas al hongo Coriolus versicolor. Similar a lo anterior, se encuentran los valores reportados por Donoso et al. (1984), en un estudio con la madera de las especies Prosopis tamarugo y Prosopis alba expuestas a los hongos T. versicolor y Lentinus lepideus, la pérdida de masa en su ensayo fue de $4 \%$. 


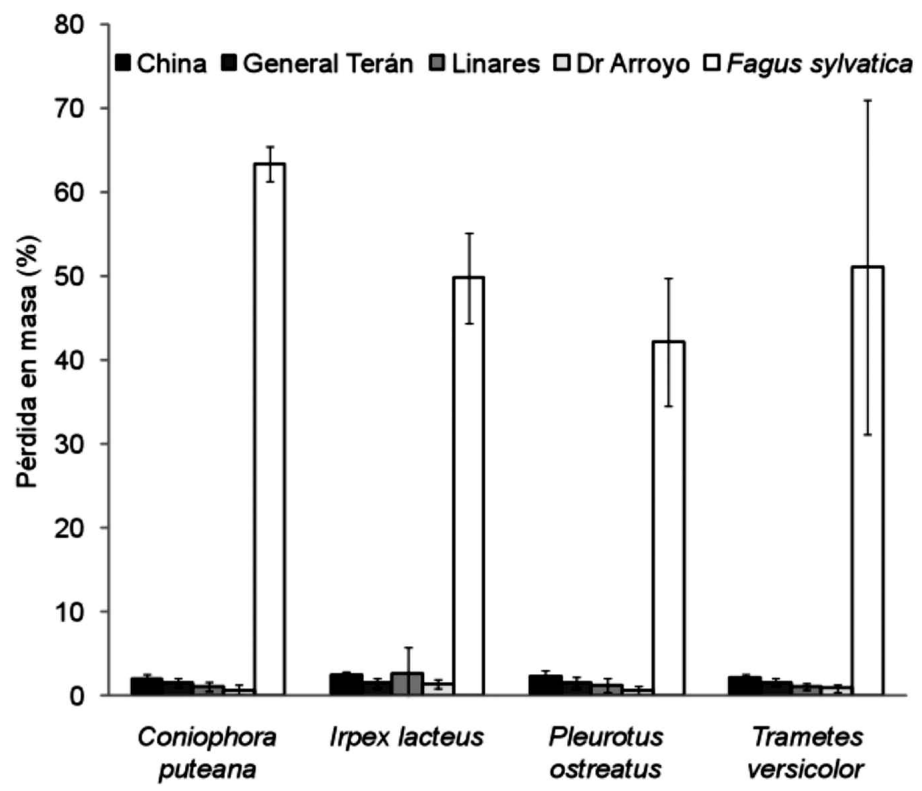

Hongos degradadores de la madera

Figura 3. Pérdida de masa de la madera de $P$. laevigata proveniente de cuatro sitios y el testigo F. sylvatica, expuestas por 16 semanas a cuatro hongos degradadores de la madera.

La durabilidad natural de $P$. laevigata puede ser el resultado de la interacción de una serie de características intrínsecas de la especie como la presencia de compuestos fenólicos en los lúmenes, punteaduras y células del parénquima que pueden proporcionar una barrera física para el paso de los micelios de los hongos a través de las estructuras anatómicas de la madera.

Por otra parte, la presencia de los compuestos catequina $(5,33 \%)$, epicatequina $(0,51 \%)$ y taxifolina $(0,05 \%)$ reportados por Carrillo et al. (2008), los primeros dos son conocidos como antioxidantes que pueden reducir la absorción de agua y por consecuencia la formación de una condición poco apropiada para el desarrollo del hongo. La taxifolina compuesto químico que también ha sido encontrado en la madera de los géneros Larix, Cedrus y Pseudotsuga (Eaton y Hale, 1993) y que fue considerado por Kennedy (1956) como un agente antifúngico.

\section{Pérdida del $M O E_{\text {din }}$}

Los resultados de analizar las probetas mediante la pérdida del $\mathrm{MOE}_{\text {din }}$ se encuentran en la figura 4. En esta se puede apreciar que el sitio General Terán mostró mayor reducción para todos los hongos. En la tabla 2, se destaca que el control $F$. sylvatica presenta valores altos de pérdida de $\mathrm{MOE}_{\text {din }}(67,6 \pm 7,8) \%$, $(87,2$ $\pm 9,6) \%,(80,0 \pm 9,2) \%$ y $(78,3 \pm 14,2)$ para los hongos $C$. puteana, I. lacteus, $P$. ostreatus y $T$. versicolor, respectivamente. En forma contraria, la madera de $P$. laevigata en todos los sitios y hongos presentó valores bajos. El sitio China mostró menor pudrición con un promedio 
Tabla 2. Reducción del $\mathrm{MOE}_{\text {din }}$ de las maderas de $P$. laevigata proveniente de cuatro sitios y el testigo $F$. sylvatica, expuestas a cuatro hongos degradadores de la madera.

\begin{tabular}{|c|c|c|c|c|}
\hline \multirow[b]{2}{*}{ Sitio } & \multicolumn{3}{|c|}{ Pérdida $\mathrm{MOE}_{\text {din }}(\%)$} & \multirow[b]{2}{*}{ T. versicolor } \\
\hline & C. puteana & I. lacteus & P. ostreatus & \\
\hline China & $1,4 \pm 8,0$ & $9,6 \pm 8,8$ & $0,2 \pm 5,2$ & $4,0 \pm 12,2$ \\
\hline General Terán & $18,1 \pm 34,4$ & $28,0 \pm 11,8$ & $15,0 \pm 17,0$ & $18,8 \pm 10,4$ \\
\hline Linares & $3,4 \pm 16,3$ & $9,4 \pm 6,0$ & $13,2 \pm 22,4$ & $2,0 \pm 4,8$ \\
\hline Dr. Arroyo & $4,1 \pm 23,6$ & $5,9 \pm 5,6$ & $6,2 \pm 13,6$ & $3,7 \pm 32,8$ \\
\hline F. sylvatica & $67,6 \pm 7,8$ & $87,2 \pm 9,6$ & $80,0 \pm 9,2$ & $78,3 \pm 14,2$ \\
\hline
\end{tabular}

China

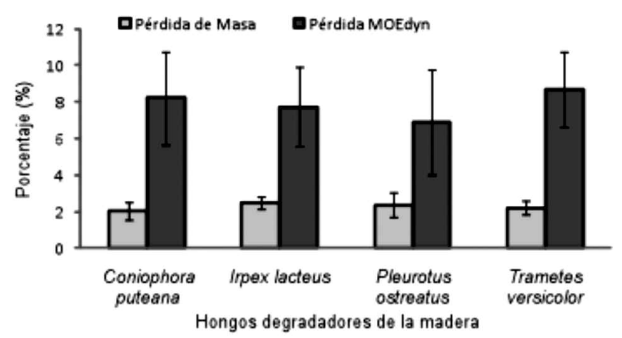

Linares

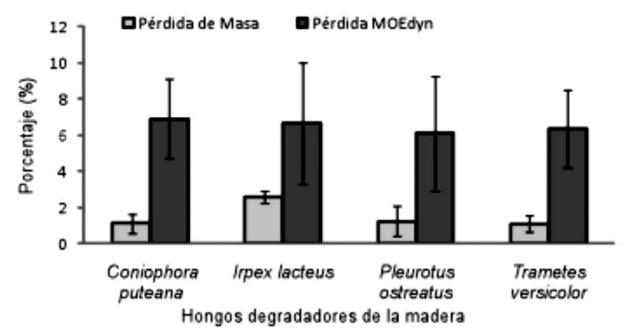

General Terán

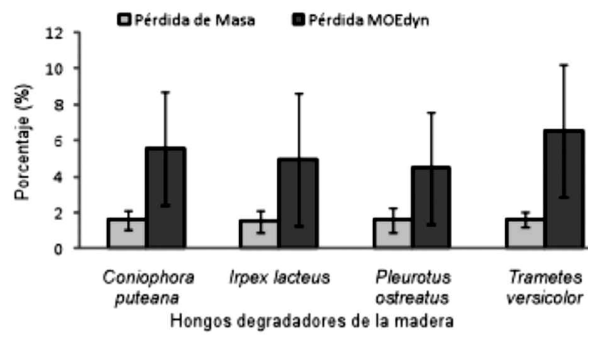

Dr. Arroyo

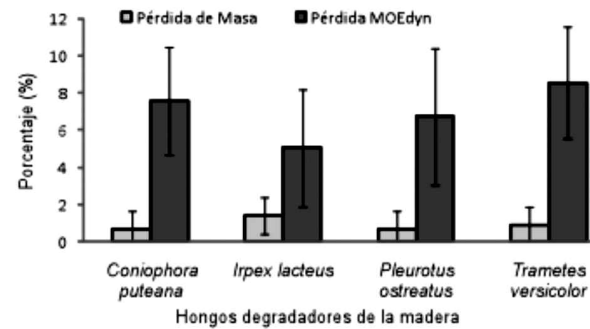

Figura 4. Valores promedio de la pérdida en masa y pérdida del $\mathrm{MOE}_{\text {dyn }}$ de la madera de $P$. laevigata proveniente de cuatro sitios al ser expuestas durante 16 semanas a cuatro hongos degradadores de la madera. 
de $3,8 \pm 4,2 \%$ para todos los hongos, seguida por los sitios Dr. Arroyo con $5,0 \pm 1,3$ y Linares con $7,0 \pm 5,2 \%$ y, finalmente, en el sitio General Terán con $19,9 \pm 5,6 \%$.

\section{Inhibición en el crecimiento de los hongos provocado por los extractos de la madera}

Los hongos C. puteana y T. versicolor, causantes de la pudrición café y pudrición blanca, respectivamente, muestran una fuerte inhibición en su crecimiento al colocarlos en un medio de cultivo con extractos de la madera de $P$. laevigata. Como se puede apreciar en la figura 5 , los extractos en una concentración de 10000 ppm obtenidos de la solubilidad con agua caliente presentan porcentajes de inhibición del crecimiento de $83 \pm 8 \%$ y $93 \pm 6 \%$ para C. puteana y $T$. versicolor, respectivamente.
Algo similar ocurre con los extractos obtenidos de etanol como solvente y en concentración de 10000 ppm. La inhibición en el crecimiento de C. puteana fue de $81 \pm 3 \%$ a $93 \pm 4 \%$ para T. versicolor. El efecto inhibitorio producido por extractos de etanol ya ha sido reportado por Sjöström (1981), Eaton y Hale (1993) y Velásquez et al. (2006), quienes coinciden al explicar que el efecto se debe a que el etanol tiene la capacidad de extraer compuestos cíclicos aromáticos, principalmente fenoles, polifenoles y lignina de bajo peso molecular, los cuales son sustancias con una fuerte influencia en la resistencia a la degradación de la madera (Eaton y Hale, 1993; Schultz y Nicholas, 1997; Singh y Kim, 1997).

Por otra parte, los extractos obtenidos utilizando acetona y en cualquiera de las cuatro concentraciones (100 ppm,

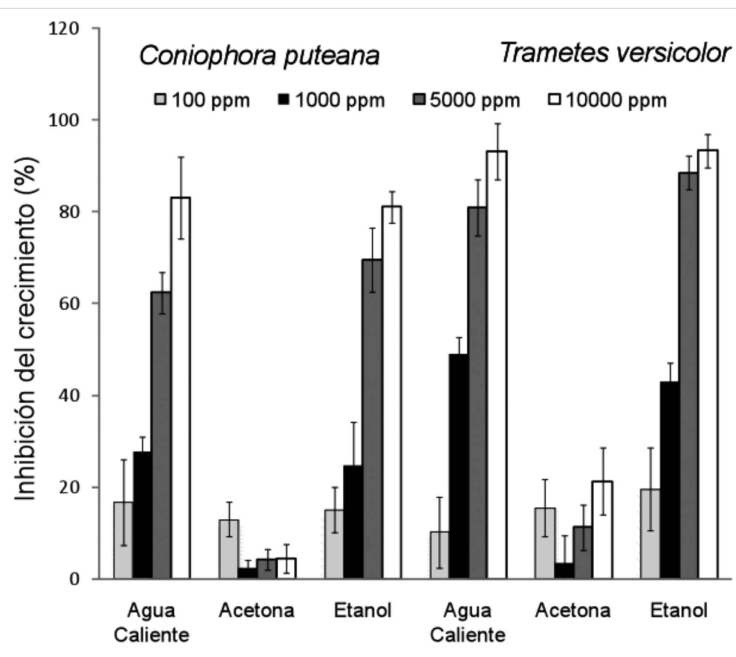

Figura 5. Inhibición del crecimiento de los hongos $C$. puteana y $T$. versicolor producido por extractos de $P$. laevigata en concentraciones de 100 ppm, 1000 ppm, 5000 ppm y 10000 ppm. 
1000 ppm, 5000 ppm y 10000 ppm) no mostraron inhibición del crecimiento para C. puteana, el rango fue de $4,4 \pm 3,1 \%$ a $12,9 \pm 15,5 \%$ y en $T$. versicolor la inhibición fue desde $3,4 \pm 6,0 \%$ hasta $21,2 \pm$ $7,2 \%$. No se encontró inhibición en el crecimiento de los hongos al añadir $10 \mathrm{ml}$ de acetona al medio de cultivo en los testigos, ya que el crecimiento de los hongos en estas cajas se presentó sin alteraciones; el uso de acetona como disolvente no estuvo influenciando los resultados reportados.

Géardin et al. (2004), reportaron en un estudio para determinar la inhibición en el crecimiento de hongos, $50 \%$ de inhibición en el crecimiento de los hongos al utilizar extractos de Prosopis africana obtenidos de la solubilidad con agua caliente y etanol en concentraciones de 1000 ppm.

\section{CONCLUSIONES}

Se encontró que la madera de $P$. laevigata proveniente de los sitios China, General Terán, Linares y Dr. Arroyo es altamente durable, al ser expuesta por 32 semanas a un microcosmos terrestre, la pérdida en masa fue menor a $17 \%$, y la mayor pérdida de $\mathrm{MOE}_{\text {din }}$ fue de $39 \%$. De igual forma, la madera de $P$. laevigata fue altamente durable al exponerla según las condiciones establecidas en la Norma Europea (EN 113) al ataque de los hongos $C$. puteana, I. lacteus, $P$. ostreatus y $T$. versicolor, causantes de la pudrición de la madera. La pérdida de masa en promedio fue menor a $2,5 \%$ para todos los hongos y la pérdida de $\mathrm{MOE}_{\text {dyn }}$ fue menor a $19 \%$. Por lo expuesto anteriormente y con base en la Norma Europea $350-1$, la madera de Prosopis laevigata es clasificada con una durabilidad Clase 1 (muy durable).
Las diferentes condiciones ambientales y la ubicación geográfica en los sitios estudiados, no representó influencia en la durabilidad de las maderas, por lo tanto pueden ser utilizadas en condiciones similares.

Los extractos de $P$. laevigata obtenidos por medio de agua caliente y etanol como solventes y en concentración a 10000 ppm, proporcionan un efecto inhibitorio en crecimiento superior a $81 \%$ para el hongo $C$. puteana y superior a $93 \%$ en $T$. versicolor. Los valores de inhibición dados por extractos obtenidos por acetona mostraron inhibiciones menores a $23 \%$ en todas las concentraciones.

\section{REFERENCIAS}

Alden, H.A. 1995. Hardwoods of North America. General Technical Report 83. USDA, Forest Service, Forest Products Laboratory. Madison, WI. $136 \mathrm{p}$.

Aloui, F., N. Ayadi, F. Charrier y B. Charrier. 2004. Durability of European oak (Quercus petraea and Quercus robur) against white rot fungi (Coriolus versicolor): relations with phenol extractives. Holz als Roh und Werkstof. 62(4):286-290.

Balaban, M., C. Atik y G. Uçar. 2003. Fungal growth inhibition by wood extracts from Juniperus foetidissima and $J$. oxycedrus. Holz als Roh und Werkstoff. 61(3):231-232.

Burkart, A. 1976. A monograph of the genus Prosopis (Leguminosae subfam Mimosoideae). Journal of the Arnold Arboretum. 57(3):219-249.

Carrillo, A., I. Mayer, G. Koch y F. Hapla. 2008. Wood anatomical characteristics and chemical composition of 
Prosopis laevigata grown in the Northeast of Mexico. IAWA Journal, Vol. 29(1):25-34.

Donoso, J., E. Cuevas, R. Rosende e I. Ulloa. 1984. Natural durability and permeability of tamarugo (Prosopis tamarugo Phil.) and algarrobo (Prosopis alba GRISEB). In, International Round Table on Prosopis tamarugo Phil. Santiago, Chile, 11 Jun 1984. FAO.

Eaton, R.A. y M.D.C. Hale. 1993. Wood: decay, pests and protection. Chapman \& Hall. London. Nueva York. $546 \mathrm{p}$.

EN 113, 1996. European Norm 113. Wood preservatives. Test method for determinating the protective effectiveness against wood destroying basidiomycetes. Determination of the toxic values. European Committe for Standarisation. Bruselas, Bélgica.

EN 350-1. 1994. European Norm 350-1. Natural Durability of Solid Wood Part 1: Guide to the Principles of Testing and Classification of the Natural Durability of Wood. European Committe for Standarisation. Bruselas, Bélgica.

ENV 807, 2001. Pre-Norma Europea 807. Wood preservatives. Determination of the effectiveness against soft rotting micro-fungi and other soil inhabiting micro-organisms. European Committe for Standarisation. Bruselas, Bélgica. ISBN 058037579 X. 44 p.

Fengel, D. y G. Wegener. 1989. Wood, chemistry, ultrastructure, reactions. Ed. Walter de Gruyter. Nueva York. EUA. 613 p.
Folliott, P.F. y J.L. Thames. 1983. Handbook on taxonomy of Prosopis in Mexico, Peru and Chile. FAO. Roma, Italia. $31 \mathrm{p}$.

Géardin, P., B. Neya, S. Dumarçay, M. Pétrissans, M. Serraj y F. Huber. 2004. Contribution of gums to natural durability of Prosopis africana heartwood. Holzforschung. 58(1):39-44.

Giménez, A.M., G. Moglia, P. Hernández y S. Bravo. 2000. Leño y la corteza de Prosopis nigra (Griseb.) Hieron, Mimosaceae, en relación a algunas magnitudes dendrométricas. Rev. Forest. Venez. 44(2):29-37.

Gutiérrez, A., V. Baonza, J.I. FernandezGolfín, M. Conde y E. Hermoso. 2006. Effect of growth conditions on wood density of spanish Pinus nigra. Wood Science and Technology. 40(3):190-204.

Haupt, M., H. Leithoff, D. Meier, J. Puls, H. G. Richter y O. Faix. 2003. Heartwood extractives and natural durability of plantation-grown teak wood (Tectona grandis L.) a case study. European Journal of Wood and Wood Products. 61(6):473-474.

Hearmon, R.F.S. 1966. Theory of the vibration testing of wood. Forest Product Journal 16(8):29-40.

Hillis, W.E. 1968. Heartwood formation and its influence on utilization. Wood Science and Technology 2(4):260267.

Hillis, W.E. 1987. Heartwood and tree exudates. Springer Series in Wood Science. Ed. Springer-Verlag. Berlín, Heidelberg, Nueva York. 268 p.

INE. 1994. Mezquite Prosopis spp. cultivo alternativo para las zonas áridas $y$ 
semiáridas de México. México. $30 \mathrm{pp}$.

Juárez M., J., G. Carrillo-C., R. Arreguín y A. Rubluo. 2002. Inter- and intragenetic variation of four wild populations of Prosopis using RAPD-PCR fingerprints. Biodiversity and Conservation 11:921-930.

Kennedy, R.W. 1956. Fungicidal toxicity of certain extraneous components of Douglas-fir heartwood. Forest Products Journal. 6:80-84.

Machek, L., H. Militz y R. Sierra-Alvarez. 1998. A dynamic approach to assess the modulus of elasticity in wood decay testing. In, Memorias International Research Group on Wood Preservation. Maastricht, Holanda.

Machek, L., H. Militz y R. Sierra-Alvarez. 2001. The use of an acoustic technique to assess wood decay in laboratory soil-bed tests. Wood Science and Technology 34(6):467-472.

Mantanis, G.I., R.A. Young y R.M. Rowell. 1995. Swelling of wood. Part III. Effect of temperature and extractives on rate and maximum swelling. Holzforschung 49(3):239-248.

Meraz, S., J. Orozco, J.A. Lechuga, F. Cruz y J. Verón. 1998. El mezquite, árbol de gran utilidad. Ciencias 51:20-21.

Pasiecznik, N.M., P. Felker, P.J.C. Harris, L.N. Harsh, G. Cruz, J.C. Tewari, K. Cadoret y L. J. Maldonado. 2001. The Prosopis juliflora-Prosopis pallida Complex: A Monograph. HIDRA, Coventry, Reino Unido.

Reyes-Chilpa, R., F. Gómez-Garibay, G. Moreno-Torres, M. Jiménez y R. I. Quiroz-Vásquez. 1998. Flavonoids and isoflavonoids with antifungal properties from Platymiscium yucatanum heartwood. Holzforschung 52(5):459-462.

Ríos, N.A., A.M. Giménez y J.G. Moglia. 2001. Crecimiento del itín (Prosopis kuntzei Harms) en la Región Chaqueña Argentina. Madera y Bosques. 7(1):47-56.

Rodríguez, F. y A. Maldonado. 1996. Overview of past, current and potential uses of mesquite in México. In: R. Felker y J. Moss, eds. Prosopis; Semiarid Fuel Wood and Forage Tree Building Consensus for the Disenfranchised. Center from Semiarid Forest Resources. Kingsville, Texas. EUA. p:6.41-6.52.

Singh, A. y Y. Kim, 1997. Biodegradation of wood in wet environments: a review. The International Research Group on Wood Preservation. Document num. IRG/WP/97-10217.

Schultz, T.P. y D.D. Nicholas. 1997. Susceptibility of angiosperm sapwood to white rot fungal colonization and subsequent degradation: a hypothesis. The International Research Group on Wood Preservation. Document num. IRG/WP/97-10211, 10 p.

Sjöström, E. 1981. Wood Chemistry: Fundamental and Applications. Academic Press Inc., Nueva York. 223 p.

Taylor, A., B.L. Gartner, J. Morrell y K. Tsunoda. 2006. Effects of heartwood extractive fractions of Thuja plicata and Chamaecyparis nootkatensis on wood degradation by termites or fungi. Journal of Wood Science. 52(2):147-153.

Thulasidas, P.K. y K.M. Bhat. 2007. Chemical extractive compounds determining the brown-rot decay resistance of teak wood. European 
Journal of Wood and Wood Products 65(2):121-124.

Velásquez, J., M.E. Toro, L. Rojas y O. Encinas. 2006. Actividad antifúngica in vitro de los extractivos naturales de especies latifoliadas de la Guayana Venezolana. Madera y Bosques 12(1):51-61.
Wolf, F. y F. Perales. 1985. Durabilidad natural de la madera de algunas especies del matorral del noreste de México. Reporte científico núm. 3. Facultad de Silvicultura y Manejo de Recursos Renovables, Universidad Autónoma de Nuevo León. UANL. ISSN 01856332. $15 \mathrm{p}$. y Bosques 17(1):7-21. 